PROCEEDINGS OF THE

AMERICAN MATHEMATICAL SOCIETY

Volume 125, Number 2, February 1997, Pages 447-454

S 0002-9939(97)03594-6

\title{
LOCAL JORDAN *-DERIVATIONS OF STANDARD OPERATOR ALGEBRAS
}

\author{
LAJOS MOLNÁR AND PETER ŠEMRL
}

(Communicated by Palle E. T. Jorgensen)

\begin{abstract}
We prove that on standard operator algebras every local Jordan *-derivation is a Jordan *-derivation.
\end{abstract}

There is an extensive study of algebraic reflexivity of certain subsets of linear transformations on vector spaces and algebras (e.g. [1], [2], [6], [8] and see also the references therein). Its main problem is concerned with the question of how the transformations under consideration are determined by their local actions. As an illustration we mention the following fundamental theorem of Larson and Sourour. Let $\mathcal{B}(X)$ denote the algebra of all bounded linear operators on the complex Banach space $X$. If $\varphi$ is a so-called local derivation of $\mathcal{B}(X)$, that is, a linear mapping from $\mathcal{B}(X)$ into itself with the property that for every $A \in \mathcal{B}(X)$ there exists a derivation $\theta_{A}$ of $\mathcal{B}(X)$ such that $\varphi(A)=\theta_{A}(A)$, then $\varphi$ is a derivation.

The aim of this paper is to contribute to this study by presenting a result of the same spirit for a new class of transformations. Our main objects are the Jordan *-derivations. If $\mathcal{B}$ is a ${ }^{*}$-ring and $\mathcal{A} \subset \mathcal{B}$ is a subring, then the additive function $J: \mathcal{A} \rightarrow \mathcal{B}$ is called a Jordan *-derivation if

$$
J\left(T^{2}\right)=T J(T)+J(T) T^{*} \quad(T \in \mathcal{A}) .
$$

It is easy to verify that for every $A \in \mathcal{B}$, the mapping $J$ defined by $J(T)=T A-$ $A T^{*} \quad(T \in \mathcal{A})$ is a Jordan *-derivation. If $A \in \mathcal{A}$, this is called an inner Jordan *-derivation. The importance of these mappings relies upon the fact that their structure plays an essential role in the problem of representability of quadratic functionals by sesquilinear forms on modules [10], [11].

However, to point out a significant difference between our result below and the other works on this field, we emphasize that although the underlying structure is an algebra and real-linearity would be reasonable to assume, the involved mappings are supposed to be merely additive. Investigations of this kind concerning isomorphisms and derivations of semi-simple Banach algebras were initiated by Kaplansky [7] and then followed, for example, by Johnson and Sinclair [5].

Received by the editors August 4, 1995.

1991 Mathematics Subject Classification. Primary 47B47, 47D25.

Key words and phrases. Standard operator algebra, Jordan *-derivation, local Jordan *derivation.

The first author was partially supported by the Hungarian National Research Science Foundation, and the second author was supported by a grant from the Ministry of Science and Technology of Slovenia. 
We begin with the notation. If $H$ is a Hilbert space, then let $\mathcal{B}(H)$ denote the algebra of all bounded linear operators on $H$ and let $\mathcal{S}(H)$ be the set of self-adjoint elements of $\mathcal{B}(H) . \mathcal{F}(H)$ stands for the algebra of bounded finite-rank operators on $H$. A subalgebra of $\mathcal{B}(H)$ which contains $\mathcal{F}(H)$ is called a standard operator algebra.

If $H$ is complex and $\mathcal{A}$ is a standard operator algebra, then [12, Theorem] together with a direct computation in the case of $\operatorname{dim} H=1$ implies that the additive mapping $J: \mathcal{A} \rightarrow \mathcal{B}(H)$ is a Jordan *-derivation if and only if there exists an $A \in \mathcal{B}(H)$ such that

$$
J(T)=T A-A T^{*} \quad(T \in \mathcal{A}) .
$$

The additive mapping $J: \mathcal{A} \rightarrow \mathcal{B}$ is said to be a local Jordan *-derivation [locally inner Jordan *-derivation] if for every $T \in \mathcal{A}$ there exists a Jordan *-derivation $J_{T}: \mathcal{A} \rightarrow \mathcal{B}$ [an element $S_{T} \in \mathcal{A}$ ] such that $J(T)=J_{T}(T)\left[J(T)=T S_{T}-S_{T} T^{*}\right]$.

Our main result is formulated in the following theorem.

Theorem. Let $H$ be a complex Hilbert space, $\operatorname{dim} H>1$ and suppose that $\mathcal{A} \subset$ $\mathcal{B}(H)$ is a standard operator algebra. Then every local Jordan *-derivation from $\mathcal{A}$ into $\mathcal{B}(H)$ is a Jordan *-derivation.

In the proof we shall need the next proposition.

Lemma. Let $J: \mathcal{A} \rightarrow \mathcal{B}(H)$ be an additive mapping with the property that for every $T \in \mathcal{A}$ there is a self-adjoint operator $A_{T} \in \mathcal{B}(H)$ such that $J(T)=T A_{T}-A_{T} T^{*}$. Assume that $P$ is an (orthogonal) projection of finite rank, $t, s \in \mathbb{R} \backslash\{0\}$ and $A, B \in \mathcal{S}(H)$ satisfy

$$
J(t P)=t(P A-A P) \quad \text { and } \quad J(i s P)=i s(P B+B P) .
$$

Then $P A-A P=P B-B P$.

Proof of Lemma. If $P=I$, there is nothing to prove. So exclude this possibility and let

$$
P=\sum_{i=1}^{n} e_{i} \otimes e_{i},
$$

where $\left\{e_{i}\right\}_{i=1}^{n}$ is an orthonormal system of vectors. Let $e \in H$ be a unit vector which is orthogonal to this system and for an $1 \leq k \leq n$ define

$$
Q=e_{k} \otimes e, \quad R=e \otimes e_{k} .
$$

Suppose that $C, D \in \mathcal{S}(H)$ are such that

$$
J(R)=R C-C Q, \quad J(i R)=i(R D+D Q) .
$$

Choose nonzero rational numbers $\lambda_{1}, \lambda_{2}, \mu_{1}, \mu_{2}$. The additivity of $J$ implies that there exist $X, Y \in \mathcal{S}(H)$ for which

$$
\begin{aligned}
& \lambda_{1} t(P A-A P)+i \mu_{1} s(P B+B P)+\lambda_{2}(R C-C Q)= \\
& \lambda_{1} t(P X-X P)+i \mu_{1} s(P X+X P)+\lambda_{2}(R X-X Q)
\end{aligned}
$$

and

$$
\begin{aligned}
& \lambda_{1} t(P A-A P)+i \mu_{1} s(P B+B P)+i \mu_{2}(R D+D Q)= \\
& \lambda_{1} t(P Y-Y P)+i \mu_{1} s(P Y+Y P)+i \mu_{2}(R Y+Y Q) .
\end{aligned}
$$


First consider equation (1). If we take the operators on both sides at $e_{k}$ and then form inner product with $e_{k}$, we obtain

$$
\left\langle B e_{k}, e_{k}\right\rangle=\left\langle X e_{k}, e_{k}\right\rangle .
$$

Applying the same operators on $e$ and calculating the inner product of the obtained vectors with $e_{k}$ we arrive at

$$
\begin{aligned}
& \lambda_{1} t\left\langle A e, e_{k}\right\rangle+i \mu_{1} s\left\langle B e, e_{k}\right\rangle-\lambda_{2}\left\langle C e_{k}, e_{k}\right\rangle \\
& \quad=\lambda_{1} t\left\langle X e, e_{k}\right\rangle+i \mu_{1} s\left\langle X e, e_{k}\right\rangle-\lambda_{2}\left\langle X e_{k}, e_{k}\right\rangle .
\end{aligned}
$$

Taking the operators in (1) once again at $e$ and then forming another inner product, this time with $e$, we get

$$
\left\langle C e, e_{k}\right\rangle-\left\langle C e_{k}, e\right\rangle=\left\langle X e, e_{k}\right\rangle-\left\langle X e_{k}, e\right\rangle .
$$

From (1-1) and (1-2) we have

$$
\left\langle X e, e_{k}\right\rangle=\frac{\lambda_{1} t\left\langle A e, e_{k}\right\rangle+i \mu_{1} s\left\langle B e, e_{k}\right\rangle-\lambda_{2}\left\langle C e_{k}, e_{k}\right\rangle+\lambda_{2}\left\langle B e_{k}, e_{k}\right\rangle}{\lambda_{1} t+i \mu_{1} s} .
$$

The self-adjointness of $C$ and $X$ together with (1-3) and (1-4) implies that

$$
\operatorname{Im}\left\langle C e, e_{k}\right\rangle=\operatorname{Im} \frac{\lambda_{1} t\left\langle A e, e_{k}\right\rangle+i \mu_{1} s\left\langle B e, e_{k}\right\rangle-\lambda_{2}\left\langle C e_{k}, e_{k}\right\rangle+\lambda_{2}\left\langle B e_{k}, e_{k}\right\rangle}{\lambda_{1} t+i \mu_{1} s} .
$$

Since this relation holds for every $0 \neq \lambda_{2} \in \mathbb{Q}$, thus, sending $\lambda_{2}$ to 0 we have

$$
\operatorname{Im}\left\langle C e, e_{k}\right\rangle=\operatorname{Im} \frac{\lambda_{1} t\left\langle A e, e_{k}\right\rangle+i \mu_{1} s\left\langle B e, e_{k}\right\rangle}{\lambda_{1} t+i \mu_{1} s} .
$$

Applying the same method to equation (2), we obtain

$$
\operatorname{Re}\left\langle D e, e_{k}\right\rangle=\operatorname{Re} \frac{\lambda_{1} t\left\langle A e, e_{k}\right\rangle+i \mu_{1} s\left\langle B e, e_{k}\right\rangle}{\lambda_{1} t+i \mu_{1} s} .
$$

It follows from (1-5) and (2-1) that the value of

$$
\frac{\lambda_{1} t\left\langle A e, e_{k}\right\rangle+i \mu_{1} s\left\langle B e, e_{k}\right\rangle}{\lambda_{1} t+i \mu_{1} s}
$$

does not depend on $\lambda_{1}$ and $\mu_{1}$, which implies

$$
\left\langle A e, e_{k}\right\rangle=\left\langle B e, e_{k}\right\rangle \text {. }
$$

The validity of the equation $P A-A P=P B-B P$ is now easy to check.

Proof of Theorem. Let $J$ be a local Jordan *-derivation on $\mathcal{A}$. Then $J$ is a linear combination of two local Jordan *-derivation having the "self-adjointness property" described in the assumptions of Lemma. More precisely,

$$
J=(1 / 2)\left(D_{1}-i D_{2}\right)
$$

where $D_{1}$ and $D_{2}$ are local Jordan *-derivations defined by

$$
D_{1}(T)=J(T)-J(T)^{*} \quad \text { and } \quad D_{2}(T)=i\left(J(T)+J(T)^{*}\right) \quad(T \in \mathcal{A})
$$

having the additional property that for every $T \in \mathcal{A}$ there is a self-adjoint operator $A_{i}$ such that $D_{i}(T)=T A_{i}-A_{i} T^{*}, \quad i=1,2$. Hence, it is enough to prove our result only for such local Jordan *-derivations.

Therefore, assume from now on that this "self-adjointness property" holds for $J$. In particular, $J(S)$ is a skew-Hermitian operator for every self-adjoint $S \in \mathcal{A}$. We shall prove that $J$ is real-linear on $\mathcal{S F}(H)$, the set of all bounded self-adjoint finite-rank operators on $H$. 
Let $P$ be a finite-rank projection and $0 \neq t_{0} \in \mathbb{R}$. There exist $A, B, C \in \mathcal{S}(H)$ such that

$$
J(P)=P A-A P, \quad J(i P)=i(P B+B P), \quad \text { and } \quad J\left(t_{0} P\right)=t_{0}(P C-C P) .
$$

Using Lemma with $t=s=1$, we get $P A-A P=P B-B P$, and once again with $t=t_{0}, \quad s=1$, we have $P C-C P=P B-B P$. It follows that

$$
J\left(t_{0} P\right)=t_{0} J(P),
$$

which holds for $t_{0}=0$ as well. Since every operator in $\mathcal{S F}(H)$ is a real-linear combination of finite-rank projections, it follows from (3) that the restriction of $J$ to $\mathcal{S F}(H)$ is real-linear.

Define a real-linear mapping $\phi: \mathcal{S F}(H) \rightarrow \mathcal{B}(H \oplus H)$ by

$$
\phi(T)=\left[\begin{array}{cc}
T & J(T) \\
0 & T
\end{array}\right] \quad(T \in \mathcal{S F}(H)) .
$$

It is easy to see that $\phi(P)^{2}=\phi(P)$ holds for every finite-rank projection $P$. Pick an arbitrary operator $T \in \mathcal{S F}(H)$. Then $T=\sum_{i=1}^{n} t_{i} P_{i}$, where $t_{i} \in \mathbb{R}$ and $P_{i}$ is a finite-rank projection such that $P_{i} P_{j}=P_{j} P_{i}=0$ if $i \neq j$. Since $P_{i}+P_{j}$ is a projection if $i \neq j$, we have $\left(\phi\left(P_{i}\right)+\phi\left(P_{j}\right)\right)^{2}=\phi\left(P_{i}\right)+\phi\left(P_{j}\right)$. This yields $\phi\left(P_{i}\right) \phi\left(P_{j}\right)+\phi\left(P_{j}\right) \phi\left(P_{i}\right)=0$. Using this relation it is easy to see that $\phi(T)^{2}=\phi\left(T^{2}\right)$ which further implies

$$
J\left(T^{2}\right)=T J(T)+J(T) T \quad(T \in \mathcal{S F}(H)) .
$$

Linearizing this equation, i.e. replacing $T$ by $T+S$, we obtain

$$
J(T S+S T)=T J(S)+S J(T)+J(T) S+J(S) T \quad(T, S \in \mathcal{S F}(H)) .
$$

Next, we define a complex-linear mapping $J_{1}: \mathcal{F}(H) \rightarrow \mathcal{F}(H)$ by

$$
J_{1}(R)=J_{1}(T+i S)=J(T)+i J(S) \quad(R \in \mathcal{F}(H)),
$$

where $T=(1 / 2)\left(R+R^{*}\right)$ is the real part and $S=(2 i)^{-1}\left(R-R^{*}\right)$ is the imaginary part of $R$, respectively. Applying (4) it is easy to see that $J_{1}$ is a Jordan derivation, that is,

$$
J_{1}\left(R^{2}\right)=R J_{1}(R)+J_{1}(R) R \quad(R \in \mathcal{F}(H)) .
$$

Using a result of Herstein [4, Theorem 3.1] we infer that $J_{1}$ is a derivation and then, by a well-known theorem of Chernoff [3, Corollary 3.4], we conclude that there exists an $A \in \mathcal{B}(H)$ such that

$$
J_{1}(R)=R A-A R \quad(R \in \mathcal{F}(H)) .
$$

Clearly, we have $J_{1}(S)=J(S)$ for every $S \in \mathcal{S F}(H)$. It follows that $S A-A S$ is a skew-Hermitian operator whenever $S \in \mathcal{S F}(H)$. A straightforward computation shows that $A-A^{*}$ commutes with every element of $\mathcal{S F}(H)$ and this implies that $A-A^{*}=i t I$ for some real number $t$. The relation (5) holds true also in the case when we replace $A$ by $A-(1 / 2) i t I$. Hence, we can assume with no loss of generality that the operator $A$ in (5) is self-adjoint.

Now let us define an additive maping $J_{2}: \mathcal{A} \rightarrow \mathcal{B}(H)$ by

$$
J_{2}(R)=J(R)-\left(R A-A R^{*}\right) \quad(R \in \mathcal{A}) .
$$

This is obviously a local Jordan *-derivation having the "self-adjointness property". Moreover, $J_{2}(S)=0$ for all $S \in \mathcal{S F}(H)$. We are going to show that $J_{2}$ is a Jordan *-derivation on $\mathcal{F}(H)$. 
Let $e, f \in H$ be mutually orthogonal unit vectors and define projections $Q_{s}, \quad 0 \leq$ $s \leq 1$, and $R_{k}, k=0,1$, by

$$
\begin{aligned}
& Q_{s}=\left(s^{1 / 2} e+(1-s)^{1 / 2} f\right) \otimes\left(s^{1 / 2} e+(1-s)^{1 / 2} f\right), \\
& R_{k}=(1 / 2)\left(e+(-1)^{k} f\right) \otimes\left(e+(-1)^{k} f\right) .
\end{aligned}
$$

Suppose that $0 \neq t \in \mathbb{R}$ and let $P$ and $B$ be a rank-one projection and a selfadjoint operator, respectively. Assume that $J_{2}(i t P)=i t(P B+B P)$. It follows from Lemma and $J_{2}(P)=0$ that $B$ commutes with $P$ and thus $J_{2}(i t P)$ is a real scalar multiple of $i P$. Hence, there exists an additive function $f: \mathbb{R} \rightarrow \mathbb{R}$ such that $J_{2}(i t P)=i f(t) P(t \in \mathbb{R})$. Similarly, we can find additive functions $f_{s}, g_{k}: \mathbb{R} \rightarrow \mathbb{R}$, $0 \leq s \leq 1, k=0,1$, satisfying

$$
J_{2}\left(i t Q_{s}\right)=i f_{s}(t) Q_{s} \quad \text { and } \quad J_{2}\left(i t R_{k}\right)=i g_{k}(t) R_{k} \quad(t \in \mathbb{R})
$$

for every $0 \leq s \leq 1, k=0,1$. We have $R_{1}=Q_{0}+Q_{1}-R_{0}$ and consequently,

$$
\begin{aligned}
g_{1}(t) Q_{0}+g_{1}(t) Q_{1}-g_{1}(t) R_{0} & =-i J_{2}\left(i t R_{1}\right) \\
& =f_{0}(t) Q_{0}+f_{1}(t) Q_{1}-g_{0}(t) R_{0} \quad(t \in \mathbb{R}),
\end{aligned}
$$

which implies that

$$
f_{0}=f_{1}=g_{0}=g_{1}
$$

Moreover, we have

$$
\begin{aligned}
i t Q_{s}=i\left(2 t\left(s-s^{2}\right)^{1 / 2} R_{0}\right. & +\left(t(1-s)-t\left(s-s^{2}\right)^{1 / 2}\right) Q_{0} \\
& \left.+\left(t s-t\left(s-s^{2}\right)^{1 / 2}\right) Q_{1}\right) \quad(t \in \mathbb{R}, \quad 0 \leq s \leq 1)
\end{aligned}
$$

and it follows that

$$
\begin{aligned}
f_{s}(t) Q_{s}= & g_{0}\left(2 t\left(s-s^{2}\right)^{1 / 2}\right) R_{0}+f_{0}\left(t(1-s)-t\left(s-s^{2}\right)^{1 / 2}\right) Q_{0} \\
& +f_{1}\left(t s-t\left(s-s^{2}\right)^{1 / 2}\right) Q_{1}
\end{aligned}
$$

whenever $t \in \mathbb{R}, 0 \leq s \leq 1$. Taking the operators on both sides at $e$ and $f$ and forming inner product with $e$ respectively $f$, we obtain

$$
f_{s}(t) s=g_{0}\left(t\left(s-s^{2}\right)^{1 / 2}\right)+f_{1}\left(t s-t\left(s-s^{2}\right)^{1 / 2}\right),
$$

and

$$
f_{s}(t)(1-s)=g_{0}\left(t\left(s-s^{2}\right)^{1 / 2}\right)+f_{0}\left(t(1-s)-t\left(s-s^{2}\right)^{1 / 2}\right)
$$

for every $t \in \mathbb{R}$ and $0 \leq s \leq 1$. As a consequence of (6) we have

$$
\frac{f_{1}(t s)}{s}=\frac{f_{1}(t(1-s))}{1-s}=\frac{f_{1}(t)-f_{1}(t s)}{1-s} \quad(t \in \mathbb{R}, 0<s<1) .
$$

Hence

$$
f_{1}(t s)=f_{1}(t) s \quad(t \in \mathbb{R}, 0<s<1) .
$$

Replacing $t$ by 1 we get

$$
f_{1}(s)=\text { as } \quad(0<s<1),
$$

where $a=f_{1}(1)$. Since $f_{1}$ is additive, this last equation holds for every real number $s$.

Let us now assume that $\operatorname{dim} H \geq 3$ and let $K$ and $L$ be arbitrary projections of rank one. Just as before, we obtain that there are real numbers $a_{1}$ and $a_{2}$ such that 
$J_{2}(i t K)=i a_{1} t K$ and $J_{2}(i t L)=i a_{2} t L$ hold for every real $t$. We can find a projection $M$ having rank one and being orthogonal to $K$ and $L$. We have $J_{2}(i t M)=i a_{3} t M$ $(t \in \mathbb{R})$, where $a_{3}$ is a fixed real number. From the discussion above it follows that $a_{1}=a_{3}$ and $a_{2}=a_{3}$. Hence, there exists a real constant $a$ such that for every projection $P$ with rank one we have $J_{2}(i t P)=i a t P$. But this implies that

$$
J_{2}(T)=a T
$$

for every skew-Hermitian finite-rank operator $T$. Thus

$$
J_{2}(T)=T((a / 2) I)-((a / 2) I) T^{*}
$$

holds for every finite-rank operator $T$. Therefore, the restriction of $J$ to $\mathcal{F}(H)$ is a Jordan *-derivation.

The proof in this case can be completed as it was done in $[8$, Proof of Theorem 1.2]. That is, by [12, Theorem], there is an operator $A \in \mathcal{B}(H)$ such that $J(T)=$ $T A-A T^{*}$ for every $T \in \mathcal{F}(H)$. Let

$$
J_{3}(T)=J(T)-\left(T A-A T^{*}\right) \quad(T \in \mathcal{A}) .
$$

We have to show that $J_{3}=0$. Let $T \in \mathcal{A}, x \in H$ and let $P$ denote the orthogonal projection onto the subspace generated by the vectors $x, J_{3}(T) x$. Since $J_{3}$ is a local Jordan *-derivation which vanishes on $\mathcal{F}(H)$, we have

$$
0=P J_{3}((I-P) T(I-P)) P=P J_{3}(T) P
$$

and this implies $J_{3}(T) x=0$.

Finally, in the two-dimensional case we identify $\mathcal{B}(H)$ with the algebra of all $2 \times 2$ complex matrices. We have already proved that there is a real number $a$ such that

$$
J_{2}\left(i t Q_{0}+i s Q_{1}\right)=a\left(i t Q_{0}+i s Q_{1}\right) \quad(t, s \in \mathbb{R}) .
$$

This gives together with $J_{2}\left(i t R_{0}\right)=i a t R_{0}$ that

$$
J_{2}\left(\left[\begin{array}{cc}
0 & i t \\
i t & 0
\end{array}\right]\right)=a\left[\begin{array}{ll}
0 & i t \\
i t & 0
\end{array}\right]
$$

holds for every $t \in \mathbb{R}$. Using considerations similar to those which led to (6) but replacing $R_{0}$ and $R_{1}$ by $\tilde{R}_{0}=(1 / 2)(i e+f) \otimes(i e+f)$ and $\tilde{R}_{1}=(1 / 2)(i e-f) \otimes(i e-f)$, respectively, we have $J_{2}\left(i t \tilde{R}_{0}\right)=i a t \tilde{R}_{0}$. This implies that

$$
J_{2}\left(\left[\begin{array}{cc}
0 & t \\
-t & 0
\end{array}\right]\right)=a\left[\begin{array}{cc}
0 & t \\
-t & 0
\end{array}\right]
$$

holds for every real number $t$. Thus, we have proved also in this case that $J_{2}(T)=$ $a T$ for every skew-Hermitian operator $T$. Now, one can complete the proof just as in the previous case.

Remark. As for the remaining case $\operatorname{dim} H=1$, we assert that the theorem does not hold true in this case. To see this, let $f: \mathbb{R} \rightarrow \mathbb{R}$ be any non-continuous additive function. Then the function $J: \mathbb{C} \rightarrow \mathbb{C}$ defined by

$$
J(x+i y)=i f(y) \quad(x, y \in \mathbb{R})
$$


is a local Jordan *-derivation which is not a Jordan *-derivation. If $\operatorname{dim} H=1$, the conclusion of the statement remains valid under the stronger assumption that $J$ is a real-linear local Jordan *-derivation.

We also show that in our theorem the condition that $\mathcal{A}$ contains $\mathcal{F}(H)$ cannot be omitted. In fact, let $H=\mathbb{C} \oplus \mathbb{C}$,

$$
P=\left[\begin{array}{ll}
1 & 0 \\
0 & 0
\end{array}\right]
$$

and $\mathcal{A}=\mathbb{C} P$. Take arbitrary $2 \times 2$ matrices $A, B$ and define $J: \mathcal{A} \rightarrow \mathcal{B}(H)$ by

$$
J((\lambda+i \mu) P)=\lambda(P A-A P)+i \mu(P B+B P) \quad(\lambda, \mu \in \mathbb{R}) .
$$

It is easy to see that $J$ is additive and for every $T \in \mathcal{A}$ there is a matrix $X$ such that

$$
J(T)=T X-X T^{*}
$$

which implies that $J$ is a local Jordan *-derivation. But considering the equation characterizing the Jordan *-derivations, one can check that $J$ is a Jordan *-derivation if and only if $P A-A P=P B-B P$ which obviously does not hold in general.

To conclude the paper we formulate the following analogues of the results in [9] for the case of Jordan *-derivations. Since the proofs can be based on arguments being very similar to those which can be found there, we omit them.

Proposition. Let $H$ be a complex separable Hilbert space. Then there are exactly three *-subalgebras of $\mathcal{B}(H)$ on which every Jordan *-derivation is locally inner, namely $\{0\}, \mathcal{F}(H)$ and $\mathcal{B}(H)$. Moreover, if $\mathcal{I}$ is a symmetric norm ideal in $\mathcal{B}(H)$, then every locally inner Jordan *-derivation on $\mathcal{I}$ is inner.

\section{REFERENCES}

1. M. Brešar and P. Šemrl, Mappings which preserve idempotents, local automorphisms and local derivations, Canad. J. Math. 45 (1993), 483-496. MR 94k:47054

2. M. Brešar and P. Šemrl, On local automorphisms and mappings that preserve idempotents, Studia Math. 113 (1995), 101-108.

3. P.R. Chernoff, Representations, automorphisms and derivations of some operator algebras, J. Funct. Anal. 12 (1973), 275-289. MR 50:2934

4. I.N. Herstein, Jordan derivations of prime rings, Proc. Amer. Math. Soc. 8 (1957), 1104-1110. MR 20:2362

5. B.E. Johnson and A.M. Sinclair, Continuity of derivations and a problem of Kaplansky, Amer. J. Math. 90 (1968), 1067-1073. MR 39:776

6. R.V. Kadison, Local derivations, J. Algebra 130 (1990), 494-509. MR 91f:46092

7. I. Kaplansky, Ring isomorphisms of Banach algebras, Canad. J. Math. 6 (1954), 374-381. MR 16:49e

8. D.R. Larson and A.R. Sourour, Local derivations and local automorphisms of $B(X)$, Proc. Sympos. Pure Math. 51. MR 91k:47106

9. L. Molnár, Locally inner derivations of standard operator algebras, Math. Bohem. 121 (1996), $1-7$.

10. P. Šemrl, Quadratic functionals and Jordan *-derivations, Studia Math. 97 (1991), 157-165. MR 92d:46139 
11. P. Šemrl, Quadratic and quasi-quadratic functionals, Proc. Amer. Math. Soc. 119 (1993), 1105-1113. MR 94a:15045

12. P. Šemrl, Jordan *-derivations of standard operator algebras, Proc. Amer. Math. Soc. 120 (1994), 515-518. MR 94d:46066

Institute of Mathematics, Lajos Kossuth University, H-4010 Debrecen, P.O.Box 12, HUNGARY

E-mail address: molnarl@math.klte.hu

Faculty of Technical Sciences, University of Maribor, Smetanova 17, P.O.Box 224, 62000 Maribor, Slovenia

E-mail address: peter.semrl@uni-lj.si 